\title{
ESTUDIO DE LA ALEXITIMIA Y DE LOS PROCESOS EMOCIONALES NEGATIVOS EN EL ÁMBITO DE LOS FACTORES DE RIESGO Y LA SINTOMATOLOGÍA CARDIOVASCULAR
}

\author{
BEATRIZ RUEDA Y ANA M. PÉREZ-GARCÍA \\ Facultad de Psicología, Universidad Nacional de Educación a Distancia, Madrid
}

\begin{abstract}
Resumen: Este estudio examinó las diferencias en factores de riesgo cardiovascular (hipertensión, sobrepeso, colesterol y estilo de vida no saludable), síntomas y emociones negativas (enfado manifiesto e interiorizado, rumiación y depresión) en personas con alta y baja alexitimia (AA / BA). También analizó en cada grupo cómo predecían las emociones los factores de riesgo y los síntomas. 436 participantes formaron parte de esta investigación. Los resultados indicaron que los AA llevaban un estilo de vida menos saludable que los BA, e informaban más síntomas y emociones negativas. Con respecto a los factores de riesgo no hubo diferencias. Asimismo, en los AA, el enfado manifiesto predijo negativamente la hipertensión, mientras que el enfado suprimido y la rumiación explicaron positivamente el estilo de vida. Los síntomas sólo fueron explicados por la depresión. En conclusión la alexitimia, junto con ciertas emociones nocivas, pueden influir negativamente sobre la salud y la prevención cardiovascular.
\end{abstract}

Palabras clave: Alexitimia, emociones negativas, riesgo cardiovascular, síntomas.

Relationship between alexithymia and negative emotional processes with cardiovascular risk factors and symptoms

\begin{abstract}
This study examines differences in cardiovascular risk factors (hypertension, overweight, cholesterol and unhealthy lifestyle), symptoms and negative emotions (anger-out, anger-in, rumination and depression) in people with high and low alexithymia (HA / LA). It also analyses in each group how emotions predict risk factors and symptoms. Four hundred and thirty-six participants took part in this investigation. The results indicate that HA had lifestyles less unhealthy than LA, and reported more symptoms and negative emotions. There were no differences in risk factors. Furthermore, in HA, anger-out negatively predicted hypertension, whereas anger-in and rumination positively explained the lifestyle. The symptoms could only be explained by depression. We conclude that alexithymia, together with certain noxious emotions, can negatively influence health and cardiovascular prevention
\end{abstract}

Keywords: Alexithymia, negative emotions, cardiovascular risk, symptoms.

\section{INTRODUCCIÓN}

El concepto de alexitimia, introducido en los años 70 por Nemiah y Sifneos (1970), ha ido recibiendo un gran interés dentro del ámbito de la Psicología de la salud. El estudio de este constructo se desarrolló a partir de la investiga-

Recibido: 16 febrero 2007; aceptado 12 abril 2007.

Correspondencia: Beatriz Rueda, Facultad de Psicología, Universidad Nacional de Educación a Distancia (UNED), Juan del Rosal, 10, 28040 Madrid, España. Correo-e: brueda@psi.uned.es ción clínica, con pacientes psicosomáticos que presentaban una marcada incapacidad para expresar de forma verbal o simbólica sus emociones (Sifneos, 1973). Así, la alexitimia ha sido definida como una constelación de características afectivo-cognitivas que denotan un déficit en el procesamiento y la regulación de las emociones. Este déficit implica la dificultad para identificar, expresar y diferenciar los

Agradecimientos: Esta investigación fue financiada por el Ministerio de Educación y Ciencia (Proyecto I+D, SEJ200403834). 
estados emocionales de las sensaciones corporales, y la tendencia a pensar de forma concreta y utilitaria (Nemiah, Freyberger y Sifneos, 1976; Taylor, 2000).

La alexitimia se ha asociado extensamente con la aparición de trastornos psicosomáticos (Porcelli et al., 2003) y de la alimentación (Merino, Godás y Pombo, 2002); de ahí que algunos autores (Lumley, Tomakowsky y Torosian, 1997; Taylor, Bagby y Parker (1997a) propongan que esta característica de personalidad podría incrementar el nivel de susceptibilidad a otros problemas de salud y, en general, a la enfermedad.

En este sentido, la enfermedad cardiovascular representa un tipo de trastorno en torno al cual se está generando un creciente volumen de investigaciones, con el fin de examinar su posible conexión con la alexitimia. Algunos estudios han encontrado que la prevalencia de alexitimia en pacientes con enfermedad coronaria es más alta que en pacientes con otros problemas de salud, o que en individuos sanos (Fukunishi, Numata, y Hattori, 1994). Sin embargo otros resultados concluyen que la alexitimia no parece estar implicada directamente en las causas patofisiológicas de la enfermedad cardiovascular (Valkamo, Hintikka, Niskanen y Viinamäki, 2001a; Valkamo et al., 2001b). De manera que se ha propuesto (Lumley, Stettner y Wehmer, 1996b; Lumley et al., 1997), como hipótesis alternativa, que la influencia de la alexitimia sobre los trastornos cardiovasculares, podría hacerse visible a través de indicadores más indirectos, como las conductas de riesgo y los síntomas percibidos.

Atendiendo a las conductas de riesgo, hay datos que manifiestan que las personas alexitímicas cuidan menos su estado de salud, siendo proclives a abusar del alcohol o las drogas (Speranza et al., 2004), o a cumplir en menor medida los tratamientos médicos (Abramson, McClelland, Brown y Kelner, 1991). Dentro del contexto de la prevención de la enfermedad cardiovascular, el mantenimiento de un estilo de vida saludable, definido por un conjunto de conductas como llevar una dieta sana, realizar ejercicio, reducir o eliminar el consumo de tabaco y disminuir el estrés, es considerado como un factor de protección de primer orden
(Petersen et al., 2005). Relacionando esta variable con la alexitimia, cabría esperar que las personas alexitímicas llevaran un estilo de vida poco beneficioso para su salud cardiovascular. En apoyo a esta hipótesis el estudio de Helmers y Mente (1999) reveló que la alexitimia predecía positivamente el hábito de llevar una dieta poco saludable, si bien no fue significativa en la explicación de otras conductas de riesgo como el sedentarismo. Al margen de estos resultados, la investigación de la alexitimia en relación con los estilos de vida ha sido hasta el momento escasa, precisando una mayor atención.

Si se amplía el espectro del riesgo cardiovascular, surgen también los factores de riesgo tradicionales, como la hipertensión, el sobrepeso o la hipercolesterolemia (Sebregts, Falger y Bär, 2000), los cuales podrían guardar alguna relación con el déficit cognitivo-afectivo que implica la alexitimia. En el caso de la hipertensión, una enfermedad generalmente considerada como psicosomática, su asociación con la alexitimia ha sido bastante consistente (Gage y Egan, 1984; Jula, Salminen y Saarijärvi, 1999; Todarello, Taylor, James y Fanelli, 1995). Con respecto a los otros factores de riesgo, algunos resultados avalan la relación de la alexitimia con el sobrepeso (Neumann, Sollers, Thayer y Waldstein, 2004) y la obesidad (Morosin y Riva, 1997). Otros, por el contrario, no apoyan la asociación de la alexitimia con el sobrepeso (Adami, Campostano, Ravera, Leggieri y Scopinaro, 2001) o a la hipercolesterolemia (Kojima, Frasure-Smith y Lespérance, 2001; Valkamo et al., 2001b). La reflexión sobre estos datos debe ser no obstante cautelosa, en la medida en que las muestras empleadas en algunas de estas investigaciones estaban compuestas por pacientes diagnosticados ya de enfermedad cardiovascular (Kojima et al., 2001; Valkamo et al., 2001b). De modo que no es posible generalizarlos a la población sana.

Un segundo mecanismo que también podría funcionar como punto de enlace entre la alexitimia y la enfermedad cardiovascular son los síntomas autoinformados (Lumley et al.,1996b; Lumley et al., 1997). Desde un punto de vista teórico se ha sugerido que las personas alexitímicas tienden a focalizarse en las sensaciones 
que acompañan a sus estados emocionales, interpretándolas como signos de enfermedad (Taylor, Bagby y Parker, 1997b). En distintos trabajos se ha evidenciado una relación positiva entre la alexitimia y los síntomas somáticos percibidos (Deary, Scott y Wilson, 1997; Jyväsjärvi et al., 2001), incluso después de controlar el posible efecto de covariantes, como la depresión (Lumley y Norman, 1996).

Sin embargo, no todos los resultados apoyan la vinculación de la alexitimia con la presencia de síntomas físicos (Kooiman, Bolk, Rooijmans y Trisjburg, 2004; Lundh y Simonsson-Sarnecki, 2001). Incluso dentro del ámbito de la sintomatología cardiovascular esta inconsistencia también se ha hecho clara. Torosian, Lumley, Pickard y Ketterer (1997), por ejemplo, no encontraron diferencias en alexitimia entre pacientes con isquemia silenciosa y pacientes con isquemia sintomática. Incluso el grupo con isquemia silenciosa puntuó más alto en la dimensión de alexitimia referida al pensamiento orientado al exterior, en contraste con el grupo de isquemia sintomática. Igualmente, el trabajo de Sandín, Santed, Chorot y Valiente (1996a), llevado a cabo con una muestra de estudiantes, mostró que la alexitimia, globalmente considerada, no correlacionaba con los síntomas cardiovasculares registrados diariamente durante un determinado intervalo de tiempo.

A partir de esta fundamentación empírica, el presente estudio se centró en examinar la asociación de la alexitimia con los factores de riesgo cardiovascular, comprendiendo entre estos los factores tradicionales (hipertensión, sobrepeso y colesterol) así como un factor de riesgo más general, reflejado en el mantenimiento de un estilo de vida poco saludable. Asimismo, replicando estudios previos (Sandín et al., 1996a), se pretendió determinar en qué medida la alexitimia podría estar asociada con la aparición de síntomas cardiovasculares.

Para poder estudiar y comprender la relación de la alexitimia con estos criterios, se introdujeron una serie de aspectos emocionales. La presencia de ciertas emociones negativas, como la hostilidad, el enfado manifiesto o interiorizado, y la depresión, está vinculada con el inicio de la enfermedad cardiovascular (Lett et al.,
2004; Sandín, 2002), o con la aparición de factores de riesgo cardiovascular (Hogan y Linden, 2005; Kubzansky, Cole, Kawachi, Vokonas y Sparrow, 2006; Simonsick, Wallace, Blazer y Berkman, 1995).

De otra parte, aunque las personas alexitímicas discriminan poco sus estados emocionales, se ha encontrado que la alexitimia se relaciona positivamente con la depresión (Honkalampi, Hintikka, Tanskanen, Lehtonen y Viimamäki, 2000; Sandín, Chorot, Santed y Jiménez, 1996b; Valkamo et al., 2001b), la hostilidad y el enfado (Berenbaum y Irvin, 1996; Rief, Heuser y Fichter, 1996; Rueda, Pérez-García, Sanjuán y Ruiz, 2007). Resultados estos coherentes con la idea de que, en la medida en que la alexitimia comporta una dificultad para identificar y comunicar las emociones, es esperable una mayor inhibición de las respuestas emocionales negativas, y una tendencia a su rumiación o evitación (Rueda, et al., 2007).

No obstante, a excepción de los trabajos de Berenbaum e Irvin (1996) y de Honkalampi et al. (2000), en la mayoría de estos estudios la alexitimia se ha tratado como una variable continua, y, por lo tanto, no se ha llegado a examinar cómo podrían influir distintos niveles de alexitimia sobre los estados emocionales negativos; y cómo estos, a su vez, podrían llegar a predecir diferencialmente los factores de riesgo y los cardiovasculares, dependiendo del grado de alexitimia considerado.

Tomando en consideración todos estas cuestiones, el presente estudio se planteó dos objetivos. En primer lugar, abordando la alexitimia desde distintos niveles, se trató de determinar las posibles diferencias con respecto a: (a) los factores de riesgo cardiovascular, entendiendo por estos la hipertensión, el sobrepeso, el nivel de colesterol y el estilo de vida; (b) los síntomas cardiovasculares; y (c) las emociones negativas (enfado manifiesto, enfado suprimido, rumiación y depresión). En segundo lugar se analizó en qué medida el poder explicativo de estas emociones negativas podía variar, con respecto a la predicción de los factores de riesgo y los síntomas cardiovasculares, según fuera el nivel de alexitimia.

Como hipótesis se formuló que las personas con mayor grado de alexitimia tendrían más 
probabilidad de ser hipertensas y presentarían un estilo de vida menos saludable y más emociones negativas. Además se plantearon las siguientes preguntas de investigación: (1) ¿existirían diferencias significativas en sobrepeso y colesterol, y en los síntomas cardiovasculares a partir de distintos niveles de alexitimia? y (2) ¿qué emociones negativas serían predictoras de los factores de riesgo y los síntomas cardiovasculares según el nivel de alexitimia considerado?

\section{MÉTODO}

\section{Participantes}

La muestra fue extraída a partir de una población de adultos sanos, que cursaban distintas carreras universitarias. 876 personas accedieron a participar en el estudio, aunque finalmente completaron la batería de pruebas 436 participantes. De ellos 327 fueron mujeres (75\%) y 101 hombres (23\%), habiendo 8 individuos $(2 \%)$ que no indicaron el sexo. La edad media fue de 36 años, comprendiéndose el rango de edad entre los 17 y los 63 años. Los datos que se recogieron formaban parte de una investigación longitudinal basada en tres fases. Los resultados mostrados en el presente trabajo son los referidos a la primera fase.

\section{Instrumentos de evaluación}

Escala de Alexitimia de Toronto (TAS-20; Bagby, Parker, y Taylor, 1994). Este instrumento compuesto de 20 ítems mide de forma autoinformada el constructo de alexitimia, junto con las tres dimensiones de las que ésta consta: dificultad para identificar los sentimientos; dificultad para describirlos, y pensamiento orientado hacia el exterior. El rango de respuesta contenía cinco puntos, en donde 1 = Nada y $5=$ Mucho. La TAS-20 ha ofrecido una adecuada fiabilidad y validez interna (Taylor et al., 1988), y es la escala que mayoritariamente se ha utilizado para evaluar la alexitimia. En este trabajo se utilizó la puntuación global obtenida en la TAS, siendo su coeficiente de fiabilidad ( $\alpha$ de Cronbach) de 0,84 .

Factores de Riesgo Tradicionales. Para evaluar los factores de riesgo cardiovascular, la persona debía indicar en el cuestionario si era hipertenso, si tenía sobrepeso y si, a partir de un análisis reciente, su nivel de colesterol era elevado. Dichas variables fueron codificadas con un 0 cuando la respuesta era «No» y un 1 cuando la respuesta era «Sí».

Escala de Estilo de Vida. Para saber si el estilo de vida que llevaba la persona era poco saludable, se empleó una escala de 19 ítems, de los que 6 tenían una puntuación invertida. Los ítems estaban referidos al grado en que se llevaban a cabo diferentes conductas asociadas con la prevención cardiovascular (por ejemplo, comer embutidos, tomar bollería industrial, practicar ejercicio, fumar, etc.) El rango de respuesta osciló entre 1 y 4 . La puntuación total de la escala fue multiplicada por -1 para obtener una medida global de estilo de vida poco saludable, y mantener así la misma dirección que la de los factores de riesgo tradicionales. La consistencia interna de la escala en este estudio fue alta, presentando un $\alpha$ de Cronbach de 0,70.

Escala de Síntomas Cardiovasculares. Esta escala contenía 11 ítems referidos a distintos síntomas cardiovasculares. La persona, al rellenarla, debía señalar qué síntomas presentaba en ese momento, de forma que una puntuación alta significaba mayor sintomatología cardiovascular. El $\alpha$ de Cronbach obtenido por este instrumento en la investigación fue de 0,64.

Escala de Expresión del Enfado (AX; Spielberger et al., 1985). Evalúa a través de 25 ítems la forma como la persona tiende a expresar el enfado. Se compone de tres subescalas relativas al enfado manifiesto, el enfado interiorizado, y el control de la ira. En esta investigación se emplearon las dos subescalas relativas al enfado manifiesto y al enfado suprimido, ya que estos dos tipos de emociones han sido abordados en los estudios de alexitimia (Berenbaum y Irvin, 1996; Sandín et al., 1996b). La escala AX ha mostrado una consistencia interna, y una validez convergente y discriminante apropiadas (Spielberger et al., 1985). En el estudio presente la fiabilidad de la subescala de enfado manifiesto fue alta $(\alpha$ de Cronbach $=0,78)$, mientras 
que la del enfado suprimido fue menor ( $\alpha$ de Cronbach $=0,58$ ).

Escala de Disipación - Rumiación (DRS; Caprara, 1986). Esta escala evalúa la dimensión de rumiación, entendida como el deseo y la conducta de venganza ante alguna ofensa sufrida. Para Caprara, la propensión a la venganza significa que la persona continua rumiando el daño padecido. Por lo tanto, una puntuación elevada en la escala significaba un alto grado de rumiación. La DRS se compone de 20 ítems, de los que 5 son de control, aunque en este estudio se empleó una versión revisada de la escala (Bermúdez, 1993) que no incluía los ítems de control. Aun así la consistencia interna de la DRS fue muy alta $(\alpha$ de Cronbach $=$ $0,91)$.

Escala de Depresión (HADS; Zigmond y Snaith, 1983). La HADS es una escala que permite evaluar síntomas depresivos y ansiosos en población no psiquiátrica. Se compone de 14 ítems, 7 de los cuales evalúan depresión y el resto ansiedad. En la presente investigación sólo se consideró la subescala de depresión. La obtención de una puntuación alta en esta subescala representaba mayor presencia de síntomas depresivos. La escala ha sido validada en muestra española mostrando una consistencia interna y una validez de constructo discriminante excelentes (Quintana et al., 2003). En nuestro trabajo el $\alpha$ de Cronbach de la escala fue de 0,82 .

\section{Análisis de datos}

En primer lugar se categorizó la alexitimia en dos grupos, según la puntuación mediana, con el fin de determinar si había diferencias significativas entre las personas altas y bajas en alexitimia con respecto a los factores de riesgo, los síntomas y las emociones negativas. A continuación, para las variables dicotómicas de hipertensión, sobrepeso y colesterol, en las que el valor 0 significaba «no tener dicho factor»y el valor 1 indicaba «tener dicho factor», se calcularon las diferencias mediante la prueba $\chi^{2}$. En el caso de las variables continuas, es decir, estilo de vida, síntomas cardiovasculares, enfado manifiesto, enfado suprimido y depresión, se aplicó la prueba de $t$ de student. Por último, en cada uno de los grupos formados a partir de la categorización de la alexitimia (altos y bajos), se llevaron a cabo dos tipos de análisis con el objeto de examinar la capacidad predictora de las emociones negativas. Por un lado se realizaron análisis de regresión logística, para predecir la hipertensión, el sobrepeso y el colesterol; y por otro, se efectuaron análisis de regresión múltiple para predecir el estilo de vida y los síntomas. En todos los casos el nivel de significación empleado fue $p<0,05$.

\section{RESULTADOS}

Los estadísticos descriptivos de todas las variables incluidas en el estudio se muestran en la Tabla 1. En ella se puede apreciar que, en el caso de la hipertensión y el colesterol, la muestra informó mayoritariamente que no tenía ninguno de estos dos factores (moda $=0)$, mientras que sí indicó tener sobrepeso $(\operatorname{moda}=1)$.

\section{Diferencias según el nivel de alexitimia}

Para poder constatar si había diferencias en los factores de riesgo, los síntomas cardiovasculares y las emociones negativas en función de los distintos niveles de alexitimia, dicha variable fue categorizada en dos grupos. Para ello se tuvo en cuenta la puntuación mediana $(=40)$. De manera que los participantes cuya puntuación en alexitimia fue superior a 40 se incluyeron en el grupo «Altos en alexitimia» $(n=216)$, y los participantes que puntuaron igual o por debajo de 40 formaron parte del grupo «Bajos en alexitimia» $(n=220)$.

Por una parte no se encontraron diferencias significativas entre los dos grupos en cuanto a los factores de riesgo de hipertensión, sobrepeso y colesterol. Pero, por otra, se observó (ver Tabla 2) que los Altos en alexitimia puntuaban por encima de los Bajos en estilo de vida poco saludable $\left(t_{(434)}=4,52, p<0,001\right)$ y en síntomas cardiovasculares $\left(t_{(434)}=3,71, p<0,001\right)$. Además los Altos mostraban mayor nivel de enfado manifiesto $\left(t_{(434)}=2,71, p<0,01\right)$, enfado 
Tabla 1. Estadísticos descriptivos de la alexitimia, los factores de riesgo, los síntomas cardiovasculares y las emociones negativas en la muestra total $(N=436)$

\begin{tabular}{|c|c|c|c|c|c|}
\hline & Media $(D T)$ & Mediana & Moda & Rango real & Rango posible \\
\hline Alexitimia & $41,02(9,41)$ & 40 & 32 & $20 / 73$ & $20 / 100$ \\
\hline \multicolumn{6}{|l|}{ Factores de riesgo } \\
\hline Hipertensión ${ }^{(1)}$ & 0,06 & 0 & 0 & $0 / 1$ & $0 / 1$ \\
\hline Sobrepeso $^{(2)}$ & 0,50 & 1 & 1 & $0 / 1$ & $0 / 1$ \\
\hline Colesterol $^{(3)}$ & 0,14 & 0 & 0 & $0 / 1$ & $0 / 1$ \\
\hline Estilo de vida no saludable & $51,58(6,62)$ & 52 & 54 & $33 / 68$ & $19 / 95$ \\
\hline Síntomas cardiovasculares & $1,04(1,46)$ & 0 & 0 & $0 / 8$ & $0 / 11$ \\
\hline \multicolumn{6}{|l|}{ Emociones negativas } \\
\hline Enfado manifiesto & $17,91(4,38)$ & 17 & 18 & $10 / 35$ & $10 / 40$ \\
\hline Enfado suprimido & $13,98(3,18)$ & 14 & 14 & $7 / 25$ & $7 / 28$ \\
\hline Rumiación & $24,78(12,43)$ & 24 & 16 & $0 / 60$ & $0 / 75$ \\
\hline Depresión & $10,61(3,56)$ & 9 & 8 & $7 / 26$ & $7 / 28$ \\
\hline
\end{tabular}

Nota. (1) $N=396$; casos perdidos $=40 ; 0=$ no tener HTA $/ 1=$ tener HTA; (2) $N=426$; casos perdidos $=10 ; 0=$ no tener sobrepeso / 1 = tener sobrepeso; (3) $N=312$; casos perdidos $=124 ; 0=$ no tener colesterol $/ 1=$ tener colesterol.

Tabla 2. Comparación en factores de riesgo, síntomas cardiovasculares y emociones entre los grupos de Altos y Bajos en alexitimia

\begin{tabular}{lccc}
\hline Variables & $\begin{array}{c}\text { Altos alexitimia } \\
(n=216)\end{array}$ & $\begin{array}{c}\text { Bajos alexitimia } \\
(n=220)\end{array}$ & $p^{1}$ \\
\hline Factores de riesgo $^{2}$ & & & \\
$\quad$ Hipertensión (SI/NO) & $10 / 176$ & $12 / 198$ & n.s. \\
$\quad$ Sobrepeso (SI/NO) & $104 / 104$ & $110 / 108$ & n.s. \\
$\quad$ Colesterol (SI/NO) & $22 / 194$ & $22 / 198$ & 0,001 \\
$\quad$ Estilo de vida no saludable & $-50,17(6,27)$ & $-52,98(6,67)$ & 0,001 \\
Síntomas cardiovasculares $^{3}$ & $1,04(1,46)$ & $0,78(1,28)$ & \\
Emociones negativas $^{3}$ & & & 0,01 \\
$\quad$ Enfado manifiesto $_{\text {Enfado suprimido }}$ & $18,41(4,63)$ & $17,42(4,06)$ & 0,001 \\
$\quad$ Rumiación & $15,14(3,09)$ & $12,84(2,84)$ & 0,001 \\
Depresión & $27,61(12,23)$ & $22,00(12,02)$ & 0,001 \\
\hline
\end{tabular}

Nota. ${ }^{1}$ Probabilidad de la prueba de $\chi^{2}$ o $t$ de Student; ${ }^{2}$ Número de participantes; ${ }^{3}$ Puntuación media (DT entre paréntesis).

suprimido $\left(t_{(434)}=8.09, p<0,001\right)$, rumiación $\left(t_{(434)}=4,82, p<0,001\right)$ y depresión $\left(t_{434)}=\right.$ $6,52, p<0,001)$ que los Bajos en alexitimia.

\section{Alexitimia, emociones negativas y factores de riesgo}

En cada uno de los grupos formados se realizaron diferentes análisis de regresión para conocer la capacidad predictora de las emocio- nes negativas (enfado manifiesto, enfado suprimido, rumiación y depresión). Con los factores de riesgo de hipertensión, sobrepeso y colesterol se utilizó el análisis de regresión logística, mientras que en la explicación del estilo de vida y los síntomas se procedió al análisis de regresión múltiple.

En el grupo de participantes altos en alexitimia (ver Tabla 3) se encontró que la relación entre el enfado manifiesto y la hipertensión era significativa y negativa $(p<0,05)$. La depresión 
también se asoció positivamente con la hipertensión, aunque lo hizo a un nivel marginalmente significativo $(p=0,06)$. Ni el enfado suprimido, ni la rumiación, resultaron predictores significativos de la hipertensión. Tampoco emergió en este grupo ninguna variable emocional como predictora del sobrepeso o del colesterol.

Tabla 3. Factores asociados con la presencia de hipertensión en el grupo de Altos en alexitimia

\begin{tabular}{lccc}
\hline Variable criterio: Hipertensión & $\beta$ & $E T$ & OR \\
\hline Enfado manifiesto & $-0,23^{*}$ & 0,11 & 0,79 \\
Enfado suprimido & $-0,15$ & 0,11 & 0,85 \\
Rumiación & 0,00 & 0,02 & 1,00 \\
Depresión & $0,15^{\text {a }}$ & 0,08 & 1,16 \\
\hline
\end{tabular}

Nota. $E T=$ error típico de $\beta ; O R=$ odss ratio o tasa de probabilidad; $* p<0,05 ;{ }^{a} p=0,06$.

El enfado suprimido y la rumiación contribuyeron a explicar positivamente la variabilidad del estilo de vida poco saludable; mientras que la depresión, por su parte, predijo positivamente la presencia de síntomas cardiovasculares (ver Tabla 4).

Por lo tanto los datos pusieron de relieve que, entre las personas alexitímicas, la disminución del enfado manifiesto se asociaba con una probabilidad más alta de tener hipertensión. Además, cuanto más altos eran su tendencia a suprimir y rumiar el enfado, y su estado depre-

Tabla 4: Regresión múltiple prediciendo el estilo de vida y los síntomas cardiovasculares en el grupo de Altos en alexitimia

\begin{tabular}{llllll}
\hline Variable criterio: & & & & \\
Estilo de vida no saludable & $\beta$ & $R^{2}$ & $F$ & $g l$ \\
\hline Enfado manifiesto & 0,11 & 0,10 & $6,36^{* * *}$ & 4,215 \\
Enfado suprimido & $0,15^{*}$ & & & \\
Rumiación & $0,18^{*}$ & & & \\
Depresión & 0,03 & & & \\
\hline
\end{tabular}

Variable criterio:

\begin{tabular}{lcccc} 
Síntomas cardiovasculares & $\beta$ & $R^{2}$ & $F$ & $g l$ \\
\hline Enfado manifiesto & 0,11 & 0,11 & $7,04 * * *$ & 4,215 \\
Enfado suprimido & 0,08 & & & \\
Rumiación & 0,01 & & \\
Depresión & $0,27 * * *$ & & \\
\hline
\end{tabular}

$* * * p<0,001 ; * p<0,05$. sivo, mayor era la frecuencia de llevar un estilo de vida poco saludable y de presentar sintomatología cardiovascular respectivamente.

En el grupo de participantes Bajos en alexitimia el enfado suprimido apareció como el único predictor significativo de la hipertensión, mientras que, en el caso del sobrepeso, lo fue la depresión (ver Tabla 5).

Con respecto al colesterol ninguna emoción negativa se asoció significativamente.

Tabla 5. Factores asociados con la presencia de la hipertensión y el sobrepeso en el grupo de Bajos en alexitimia

\begin{tabular}{lccc}
\hline Variable criterio: Hipertensión , ET OR & & \\
\hline Enfado manifiesto & $-0,01$ & 0,07 & 0,98 \\
Enfado suprimido & $0,32^{* * *}$ & 0,10 & 1,38 \\
Rumiación & 0,01 & 0,02 & 1,01 \\
Depresión & $-0,00$ & 0,10 & 0,99 \\
\hline Variable criterio: Sobrepeso , ET OR & & \\
\hline Enfado manifiesto & $-0,06$ & 0,03 & 0,94 \\
Enfado suprimido & 0,03 & 0,05 & 1,04 \\
Rumiación & $-0,00$ & 0,01 & 0,99 \\
Depresión & $0,11^{*}$ & 0,05 & 1,12 \\
\hline
\end{tabular}

Nota. $E T=$ error típico de $\beta ; O R=$ odss ratio o tasa de probabilidad; *** $p<0,001 ; * p<0,05$.

Por último, el enfado manifiesto predijo positivamente el mantenimiento de un estilo de vida poco saludable, sin que el resto de emociones negativas alcanzara el nivel de significación (ver Tabla 6). Tampoco contribuyó de manera significativa ninguna de las emociones negativas en la explicación de los síntomas cardiovasculares.

Estos datos revelaron, en personas con bajo nivel de alexitimia, que la tendencia al enfado suprimido y la depresión aumentaba, respectivamente, la posibilidad de tener hipertensión y

Tabla 6. Regresión múltiple prediciendo el estilo de vida en el grupo de Bajos en alexitimia

\begin{tabular}{lcccc}
\hline Variable criterio: & & & & \\
Estilo de vida no saludable & $\beta$ & $R^{2}$ & $F$ & $g l$ \\
\hline Enfado manifiesto & $0,20 * *$ & 0,06 & $3,69 * *$ & 4,219 \\
$\quad$ Enfado suprimido & $-0,03$ & & & \\
$\quad$ Rumiación 0,08 & & & & \\
$\quad$ Depresión 0,04 & & & & \\
$* * * p<0,01$. & & & &
\end{tabular}


sobrepeso. Asimismo, la expresión abierta del enfado se asociaba con un estilo de vida poco saludable. La presencia de síntomas cardiovasculares, sin embargo, no apareció relacionada con ninguna de las respuestas emocionales estudiadas.

\section{DISCUSIÓN}

El presente estudio trató de examinar las posibles diferencias con respecto a los factores de riesgo cardiovascular, los síntomas y las emociones negativas en personas con distinto nivel de alexitimia. Asimismo se analizó en qué medida las emociones negativas contribuían a explicar tanto los factores de riesgo como los síntomas en función del nivel de alexitimia.

En consonancia con la hipótesis formulada, los resultados obtenidos pusieron de manifiesto, en primer lugar, diferencias significativas en relación con el enfado manifiesto, el enfado suprimido, la rumiación y la depresión, siendo las personas con mayor nivel de alexitimia las que puntuaron más alto en estas emociones.

Estos datos confirman tanto la idea de que la alexitimia se acompaña de un estado emocional depresivo (Honkalampi et al., 2000; Sandín et al., 1996b; Valkamo et al., 2001b); como el hecho de que la alexitimia se relaciona positivamente con distintas manifestaciones de la hostilidad, incluyendo la supresión del enfado y su consiguiente rumiación, pero también su expresión abierta. De ahí que la experiencia del enfado en la alexitimia pueda estar canalizada a través de manifestaciones no verbales para evitar su confrontación (Berenbaum y Irvin, 1996; Linden, Lenz, y Stossel, 1996), o a través de estallidos repentinos de ira cuando la dificultad para describir este sentimiento sea menor (Rueda et al., 2007).

En cuanto a las diferencias con respecto a los factores de riesgo cardiovascular tradicionales, es decir, la hipertensión, el sobrepeso y el colesterol, no se apreciaron diferencias significativas. En principio la literatura revisada ha mostrado una relación inconsistente en la relación de la alexitimia con el sobrepeso y el colesterol (Adami et al., 2001; Kojima et al., 2001), lo que iría en la línea de los datos obte- nidos en este estudio. Sin embargo, la asociación con la hipertensión ha sido más sólidamente apoyada (Gage y Egan,1984; Jula et al., 1999; Todarello et al., 1995).

Una posible explicación sobre esta cuestión podría basarse en el tipo de muestra empleada. Mientras que en este trabajo la muestra utilizada estaba compuesta por personas sanas, en los trabajos referidos las muestras elegidas estaban formadas por pacientes hipertensos. De esta forma la alexitimia también podría haber sido, no tanto una variable antecedente, como una reacción secundaria al propio diagnóstico de la hipertensión o a su dimensión crónica.

Sí se constató, por el contrario, que las personas más alexitímicas mantenían un estilo de vida menos saludable que el que seguían las personas con menor alexitimia. Al hilo de lo sugerido por distintos autores (Lumley et al., 1996b; Lumley et al., 1997), este aspecto indica que la alexitimia podría incrementar la susceptibilidad a presentar algún factor de riesgo cardiovascular o a desarrollar la enfermedad cardiovascular, mediante la realización de conductas no saludables, siendo menos probable su influencia directa sobre los cambios orgánicos.

Por otra parte, la consideración en este trabajo de las conductas de riesgo desde un punto de vista más generalizado, con el objeto de obtener una medida de estilo de vida pro-coronario, va más allá de los estudios previos en los que se ha abordado la relación de la alexitimia sólo con conductas de riesgo específicas (Helmers y Mente, 1999).

Los diferentes análisis de regresión realizados mostraron también unos resultados interesantes, en cuanto a qué variables emocionales contribuían a explicar los diferentes factores de riesgo dependiendo del nivel de alexitimia. Así, en el grupo con mayor puntuación en alexitimia, se apreció que una baja manifestación del enfado se asociaba con una mayor probabilidad de tener hipertensión; y que la tendencia a suprimir el enfado y a rumiarlo predecía en estas personas el tener un estilo de vida poco saludable.

El papel de la falta de expresión del enfado en el incremento de la presión arterial ha sido sostenido por algunas investigaciones (Hogan y Linden, 2005; Shapiro, Goldstein, y Jam- 
ner,1995), al igual que lo ha sido la influencia negativa que tiene la hostilidad sobre el cuidado de la salud (Calhoun, Bosworth, Siegler y Bastian, 2001; Miller, Markides, Chiriboga y Ray, 1995).

En el caso que nos ocupa se puede pensar que una baja manifestación del enfado acompañada de un alto nivel de alexitimia puede incrementar la posibilidad de tener hipertensión. Además, las personas con alta alexitimia tienden a manejar inadecuadamente la emoción del enfado mediante la supresión y la rumiación, lo que puede influir en llevar a cabo conductas poco saludables como posibles mecanismos de afrontamiento (Lumley, Stettner, y Wehmer, 1996a).

Por otra parte, en el grupo con menor puntuación en alexitimia se apreció que el enfado suprimido se asociaba con una mayor probabilidad de tener hipertensión, mientras que la depresión se vinculaba con el sobrepeso. En contraste con el grupo anterior, en los individuos con menor grado de alexitimia, la expresión abierta del enfado predijo un estilo de vida poco saludable

En relación con el colesterol, en ninguno de los dos grupos emergió alguna variable emocional como predictora. Por lo tanto parece que el colesterol viene a representar un factor de riesgo con el que la alexitimia no guarda relación.

Finalmente, los resultados revelaron diferencias significativas en los sintomas cardiovasculares en función del nivel de alexitimia, de manera que las personas con mayor grado de alexitimia informaron de un nivel más alto de síntomas. Estos datos, a la vez que congruentes con los presentados en otras investigaciones (Deary et al., 1997; Jyväsjärvi et al., 2001; Lumley y Norman, 1996), aportan un mayor conocimiento sobre la vinculación de la alexitimia con los síntomas de tipo cardiovascular.

Los análisis de regresión múltiple indicaron que, entre los participantes con mayor puntuación en alexitimia, la presencia de un estado emocional depresivo predecía positivamente los síntomas cardiovasculares; mientras que en el grupo de participantes con menor puntuación en alexitimia, ninguna emoción resultó predictora. Cabe suponer en este sentido que el fraca- so que comporta la alexitimia con respecto a la regulación e identificación de la experiencia afectiva, puede generar un estado de depresión y malestar, el cual se exprese a través de síntomas físicos (Taylor, Bagby, y Parker, 1997c).

El estudio realizado cuenta con una serie de limitaciones que es necesario tener en cuenta. En primer lugar, su carácter transversal no permite establecer una relación de causalidad entre la alexitimia y la aparición de los factores de riesgo cardiovascular, el tipo estilo de vida mantenido, o la sintomatología. Por ello, se hace patente la necesidad de realizar investigaciones de índole prospectiva, para determinar con mayor precisión cuál es la influencia de la alexitimia y de sus correlatos emocionales sobre los aspectos mencionados.

Además, hay que indicar que la división de la alexitimia en dos categorías no se estableció a partir del nivel clínico de alexitimia (puntuación $\geq 61$ ), ya que fueron muy pocos los participantes que obtuvieron dicha puntuación $(n=14)$. De esta forma, aun cuando los grupos formados en virtud de la puntuación mediana en alexitimia difirieron en la mayoría de las variables estudiadas, y en la capacidad predictiva de las variables emocionales, estos datos no pueden ser generalizables a otras poblaciones con un grado de alexitimia más extremo.

Por último, a la hora de examinar la relación entre la depresión y los síntomas cardiovasculares, se hace preciso controlar la posible influencia de otras variables más generales, como el neuroticismo, en la medida en que dicha relación podría ser también el reflejo de la influencia de una tendencia hacia la afectividad negativa. Y, de la misma forma, añadir que el estudio de las relaciones entre alexitimia, emociones negativas, estilo de vida y factores de riesgo clásico podría verse mejorado también con el análisis de otras variables que la literatura ha revelado importantes en el campo de la personalidad y la salud, como son las estrategias de afrontamiento, utilizadas ante el estrés o los problemas de salud (p.ej. Rueda y Pérez-García, 2005; Pelechano, 2005), que facilitan un ajuste más positivo (puede consultarse al respecto la revisión de Becoña, 2006) y podrían contribuir a la interpretación de las relaciones entre alexitimia y enfermedad física. 
En conclusión, esta investigación demuestra que la alexitimia, además de comportar un déficit en la regulación y representación de las emociones, se asocia con un peor estado de salud y con un estilo de vida poco saludable. De igual forma la alexitimia se acompaña de una constelación de emociones negativas, compuesta por el enfado manifiesto, el enfado suprimido, la rumiación y la depresión, factores todos ellos que, en unión con la alexitimia, pueden incrementar la posibilidad de desarrollar algún tipo de trastorno cardiovascular.

\section{REFERENCIAS}

Abramson, L., McClelland, D.C., Brown, D., \& Kelner, S. (1991). Alexithymic characteristics and metabolic control in diabetic and healthy adults. Journal of Nervous and Mental Disease, 179, 490-494.

Adami, G.F., Campostano, A., Ravera, G., Leggieri, M., \& Scopinaro, N. (2001). Alexithymia and body weight in obese patients. Behavioral Medicine, 27 , 121-126.

Bagby, R.M., Parker, J.D.A., \& Taylor, G.J. (1994). The twenty-item Toronto alexithymia scale-I. Item selection and cross-validation of the factor structure. Journal of Psychosomatic Research, 41, 23-32.

Becoña, E. (2006). Resiliencia: definición, características y utilidad del concepto. Revista de Psicopatología y Psicología Clínica, 11, 125-146.

Berenbaum, H., \& Irvin, S. (1996). Alexithymia, anger and interpersonal behavior. Psychotherapy and Psychosomatics, 65, 203-208.

Bermúdez, J. (1993). Adaptación española de la escala de Disipación-Rumiación: Datos preliminares. Informe del Departamento de Psicología de la Personalidad. Madrid: UNED.

Calhoun, P.S., Bosworth, H.B., Siegler, I.C., \& Bastian, L.A. (2001). The relationship between hostility and behavioral risk factors for poor health in women veterans. Preventive Medicine: An international journal devoted to practice and theory, 33, 552-557.

Caprara, G.V. (1986). Indicators of aggression: The Dissipation-Rumination Scale. Personality and Individual Differences, 6, 763-769.

Deary, I.J., Scott, S., \& Wilson, J.A. (1997). Neuroticism, alexithymia and medically unexplained symptoms. Personality and Individual Differences, 22, 551-564.

Fukunishi, I., Numata, Y., \& Hattori, M. (1994). Alexithymia and defense mechanisms in myocardial infarction. Psychological Reports, 75, 219-223.
Gage, B.C., \& Egan, K.J. (1984). The effect of Alexithymia on morbidity in hypertension. Psychotherapy and Psychosomatics, 41, 136-144.

Helmers, K.F., \& Mente, A. (1999). Alexithymia and health behaviors in health male volunteers. Journal of Psychosomatic Medicine, 47, 635-645.

Hogan, B.E., \& Linden, W. (2005). Curvilinear relationships of expressed anger and blood pressure in women but not in men. Evidence from two samples. Journal of Psychosomatic Research, 59, 97-102.

Honkalampi, K., Hintikka, J., Tanskanen, A., Lehtonen, J., \& Viinamäki, H. (2000). Depresión is strongly associated with alexithymia in the general population. Journal of Psychosomatic Research, 48, 99-104.

Jula, A., Salminen, J.K., \& Saarijärvi, S. (1999). Alexithymia: A facet of essential hypertension. Hypertension, 33, 1057-1061.

Jyväsjärvi, S., Joukamaa, M., Väisänen, E., Larivaara, P., Kivelä, S.L., \& Keinänen-Kiukaanniemi, S. (2001). Somatizing frequent attenders in primary health care. Journal of Psychosomatic Research, 50, 185-192.

Kojima, M., Frasure-Smith, N., \& Lespérance, F. (2001). Alexithymia following myocardial infarction psychometric properties and correlates of the Toronto Alexithymia Scale. Journal of Psychosomatic Research, 51, 487-495.

Kooiman, C.G., Bolk, J.H., Rooijmans, H.G.M., \& Trisjburg, R.W. (2004). Alexithymia does not predict the persistence of medically unexplained physical symptoms. Psychosomatic Medicine, 66, 224-232.

Kubzansky, L.D., Cole, S.R., Kawachi, I., Vokonas, P., $\&$ Sparrow, D. (2006). Shared and unique contributions of anger, anxiety, and depression to coronary heart disease: A prospective study in the Normative Aging Study. Annals of Behavioral Medicine, 31, 1929.

Lett, H.S., Blumenthal, J.A., Babyak, M.A., Sherwood, A., Strauman, T., Robins, C., \& Newman, M.F. (2004). Depression as a risk factor for coronary artery disease: Evidence, mechanisms, and treatment. Psychosomatic Medicine, 66, 305-315.

Linden, W., Lenz, J.W., \& Stossel, C. (1996). Alexitimia, defensiveness and cardiovascular reactivity to stress. Journal of Psychosomatic Research, 41, 575-583.

Lumley, M.A., \& Norman, S. (1996). Alexithymia and health care utilization. Psychosomatic Medicine, 58, 197-202.

Lumley, M.A., Stettner, L., \& Wehmer, F.(1996a). Alexithymia, social support and health problems. Journal of Psychosomatic Research, 41, 519-530.

Lumley, M.A., Stettner, L., \& Wehmer, F.(1996b). How are alexithymia and physical illness linked?. A review and critique of pathways. Journal of Psychosomatic Research, 41, 505-518. 
Lumley, M.A., Tomakowsky, J., \& Torosian, T. (1997). The relationship to subjective and biomedical measures of disease. Psychosomatics, 38, 497-502.

Lundh, L.G., \& Simonsson-Sarnecki, M. (2001). Alexithymia, emotion, and somatic complaints. Journal of Personality, 69, 483-510.

Merino, H., Godás, A., \& Pombo, M. (2002). Alexitimia y características psicológicas asociadas a actitudes alimentarias en una muestra de adolescentes. Revista de Psicopatología y Psicología Clínica, 7, 35-44.

Miller, T., Markides, K.S., Chiriboga, D.A., \& Ray, L.A. (1995). A test of the psychosocial vulnerability and health behavior models of hostility: Results from an 11-year follow-up of Mexican Americans. Psychosomatic Medicine, 57, 572-581.

Morosin, A., \& Riva, G. (1997). Alexithymia in a clinical sample of obese women. Psychological Reports, 80, 387-394.

Nemiah, J.C., Freyberger, H., \& Sifneos, P.E. (1976). Alexithymia: A view of the psychosomatic process. En O.W. Hill (Ed.), Modern trends in psychosomatic medicine (Vol. 3, pp. 430-439). London: Butterworths

Nemiah, J.C., \& Sifneos, P.E.(1970). Affects and fantasy in patients with psychosomatic disorders. En O.W. Hill (Ed.), Modern trends in psychosomatic medicine (Vol.2, pp. 26-34). London: Butterworths.

Neumann, S.A., Sollers, J.J., Thayer, J.F., \& Waldstein, S.R. (2004). Alexithymia predicts attenuated autonomic reactivity, but prolonged recovery to anger recall in young women. International Journal of Psychophysiology, 53, 183-195.

Pelechano, V. (2005). Acerca de «afrontamiento» y «enfermedad crónica»: una reflexión ¿fundada? con alguna sugerencia. Revista de Psicopatología y Psicología Clinica, 10, 3-14.

Petersen, S., Peto, V., Rayner, M., Leal, J., Luengo-Fernández, R., \& Gray A. (2005). European cardiovascular disease statistics. www.heartstats.org.

Porcelli, P., Bagby, M., Taylor, G.J., De Carne, M., Leandro, G., \& Todarello, O. (2003). Alexithymia as predictor of treatment outcome in patients with functional gastrointestinal disorders. Psychosomatic Medicine, 65, 911-918.

Quintana, J.M., Padierna, A., Esteban, C., Arostegui, I., Bilbao, A., y Ruiz, I. ( 2003). Evaluation of the psychometric characteristics of the Spanish version of the Hospital Anxiety and Depression scale. Acta Psychiatrica Scandinavica, 107, 216-221.

Rief, W., Heuser, J., \& Fichter, M.M. (1996). What does the Toronto Alexithymia scale TAS-R measure? Journal of Clinical Psychology, 52, 423-429.

Rueda, B., y Pérez-García, A.M. (2005). Competencia percibida, procesos de valoración y afrontamiento ante un supuesto problema cardiovascular. Revista de Psicopatología y Psicología Clínica, 10, 193-203.

Rueda, B., Pérez-García, A.M., Sanjuán, P., y Ruiz, M.A. (2007). Relación entre alexitimia y hostilidad: ¿Una asociación influida por el malestar psicológico? Análisis y Modificación de Conducta, 32, 263-286.

Sandín, B. (2002). Papel de las emociones negativas en el trastorno cardiovascular: Un análisis crítico. Revista de Psicopatología y Psicología Clínica, 7, 1-18.

Sandín, B., Santed, M.A., Chorot, P., \& Valiente, R. (1996a). Alexitimia, afecto positivo y negativo, y reactividad al estrés: Relaciones con sintomatología somática y enfermedad. Análisis y Modificación de Conducta, 22, 435-460.

Sandín, B., Chorot, P., Santed, M.A., \& Jiménez, P. (1996b). Dimensiones de alexitimia y estados emocionales de ansiedad, depresión e ira. Psiquis, 17, 37-45.

Shapiro, D., Goldstein, I.B., \& Jamner, L.D. (1995). Effects of anger/hostility, defensiveness, gender, and family history of hypertension on cardiovascular reactivity. Psychophysiology, 32, 425-435.

Sebregts, E.H.W.J., Falger, P.R.J., \& Bär, F.W.H.M. (2000). Risk factor modification through nonpharmacological interventions in patients with coronary heart disease. Journal of Psychosomatic Research, 48, 425-441.

Sifneos, P.E. (1973). The prevalence of «alexythimic» characteristics in psychosomatic patients. Psychotherapy and Psychosomatics, 22, 255-262.

Simonsick, E.M., Wallace, R.B. Blazer, D.G. \& Berkman, L.F. (1995). Depressive symptomatology and hypertension-associated morbidity and mortality in older adults. Psychosomatic Medicine, 57, 427-435.

Speranza, M., Corcos, M., Stéphan, P., Loas, G., PérezDíaz, F., Lang, F., et al. (2004). Alexithymia, depressive experiences, and dependency in addictive disorders. Substance Use \& Misuse, 39, 551-579.

Spielberger, C.D., Johnson, E.H., Russell, S.F., Crane, R.J., Jacobs, G.A., \& Worden, T.J. (1985). The experience and expression of anger: Construction and validation of an anger expression scale: En M.A. Chesney, S.E. Goldston, \& R.H. Rosenman (Eds.), Anger and hostility in behavioural medicine (pp.5-30). New York: Hemisphere / McGraw.

Taylor, G.J. (2000). Recent developments in alexithymia theory and research. Canadian Journal of Psychiatry, 45, 134-142.

Taylor, G.J., Bagby, R.M., \& Parker, J.D.A. (1997a). Affects and alexithymia in medical illness and disease. En G.J. Taylor, R.M. Bagby \& J.D.A. Parker (Eds.), Disorders of affect regulation: Alexithymia in medical and psychiatric illness (pp. 216-247). Cambridge: Cambridge University Press.

Taylor, G.J., Bagby, R.M., \& Parker, J.D.A. (1997b). Affect dysregulation and alexithymia. En G.J. Taylor, R.M. 
Bagby \& J.D.A. Parker (Eds.), Disorders of affect regulation: Alexithymia in medical and psychiatric illness (pp. 26-45). Cambridge: Cambridge University Press. Taylor, G.J., Bagby, R.M., \& Parker, J.D.A. (1997c). The development and regulation of affects. En G.J. Taylor, R.M. Bagby \& J.D.A. Parker (Eds.), Disorders of affect regulation: Alexithymia in medical and psychiatric illness (pp. 7-25). Cambridge: Cambridge University Press.

Taylor, G.J., Bagby, R.M., Ryan, D.P., Parker, J.D.A., Doody, K., \& Keefe, P. (1988). Criterion validity of the Toronto Alexithymia Scale. Psychosomatic Medicine, 50, 500-509.

Todarello, O., Taylor, G.J., James, D.A., \& Fanelli, M. (1995). Alexithymia in essential hypertensive and psychiatric outpatients: A comparative study. Journal of Psychosomatic Research, 39, 987-994.
Torosian, T., Lumley, M.A., Pickard, S.D., \& Ketterer, M.W. (1997). Silent versus symptomatic myocardial ischemia: The role of psychological and medical factors. Health Psychology, 16, 123-130.

Valkamo, M., Hintikka, J., Niskanen, L., \& Viinamäki, H. (2001a). Psychiatric morbidity and the presence and absence of angiographic coronary disease in patients with chest pain. Actra Psychiatrica Scandinava, 104, 391-396.

Valkamo, M., Hintikka, J., Honkalampi, K., Niskanen, L., Koivumaa-Honkanen, H., \& Viinamäki, H. (2001b). Alexithymia in patients with coronary heart disease. Journal of Psychosomatic Research, 50, 125-130.

Zigmond, A.S., \& Snaith, R.P. (1983). The Hospital Anxiety and Depression Scale. Acta Psychiatrica Scandinavica, 67, 361-370. 